\title{
Renal replacement therapy in patients aged over 60 years
}

\author{
J.S. Tapson, R.S.C. Rodger, ${ }^{*}$ H. Mansy, R.W. Elliott, M.K. Ward and \\ R. Wilkinson
}

Department of Medicine, Freeman Hospital and Royal Victoria Infirmary, Newcastle upon Tyne NE7 7DN, UK.

\begin{abstract}
Summary: The availability of dialysis for patients with end-stage renal failure in the United Kingdom has lagged behind that in most of the rest of Europe and USA, although there has been considerable improvement over recent years. Concern about prognosis and quality of life on renal replacement therapy, together with shortage of facilities has meant that some elderly people have been denied treatment.

A retrospective study of all patients commencing renal replacement therapy in Newcastle between 1974 and 1985 was performed. The five year survival of patients aged more than 60 years at the start of treatment $(n=122)$ was $53 \%$, compared with $68 \%$ for a cohort of individuals aged less than 60 years $(n=632)$.

A questionnaire sent to the 62 elderly patients surviving at the end of the follow-up period revealed that most were married, independent, active and lived in their own home. They were not lonely, generally enjoyed life and were happy with their mode of renal replacement therapy.

These results show that elderly patients make good dialysis candidates and they should not be denied treatment on the basis of age alone. Greater funding of renal services is necessary to accommodate these patients.
\end{abstract}

\section{Introduction}

Before 1980, few patients over the age of 60 years started chronic dialysis in British renal units. Most units imposed an arbitrary age limit on the acceptance of patients for dialysis. ${ }^{1}$ The reasons for this were complex. Home haemodialysis and renal transplantation were the favoured methods of treatment. Old people, however, were rarely suitable for home haemodialysis, and there was a shortage of kidneys for transplantation. A lack of resources meant few available spaces for elderly patients in hospital haemodialysis programmes.

In 1983, Taube et al. suggested that the anticipation of a poor prognosis led to a large number of elderly patients with end-stage renal failure (ESRF) being denied proper treatment because non-nephrologists failed to refer them to renal units for assessment. ${ }^{2}$ Similar concerns about prognosis resulted in many British nephrologists practising a form of triage. ${ }^{3}$ Several groups had reported their experience of dialysis of older patients, ${ }^{4-6}$ but these studies were conducted years ago, included a high proportion of

Correspondence: J.S. Tapson B.Sc., M.R.C.P.

*Present address: Stobhill General Hospital, Glasgow, UK. Accepted: 16 July 1987 individuals aged 50-60 years, or involved only small numbers. More recent reports, ${ }^{2,7-10}$ however, have described a five year survival of dialysed elderly patients which is considerably better than that for similarly aged subjects with other potentially lethal illnesses such as certain carcinomas ${ }^{1}$ and alcoholic cirrhosis. $^{?}$

Reassurance about prognosis, and the acceptance of continuous ambulatory peritoneal dialysis (CAPD) as a particularly suitable mode of treatment ${ }^{11,12}$ has resulted in an increasing number of elderly patients with ESRF being treated in British renal units during the past 5 years.

This report describes a retrospective analysis of renal replacement therapy in patients aged over 60 years at presentation in Newcastle upon Tyne during a 12 year period between 1974 and 1985 . It is also the first paper from a British renal unit to study in-depth the quality of life experienced by the elderly patient on dialysis.

\section{Patients and methods}

One hundred and twenty two 'elderly' patients (81 males, 41 females), aged more than 60 years, commen- 
ced renal replacement therapy in Newcastle during the period between 1st January 1974 and 30th November 1985. Patient age at the start of treatment ranged from 60-76 years (mean $64.5 \pm 4.0$ years).

During the same period, 632 patients ( 376 males, 256 females) aged less than 60 years (range $1-59$ years mean $39.2 \pm 13.8$ years) were accepted for treatment of ESRF. Elderly patients, therefore, formed $16.2 \%$ of the total population. Figure 1 shows the yearly intakes and the percentages of patients who were older than 60 years at commencement of treatment.

The medical records of all patients beginning treatment for end-stage renal failure during the study period were examined. Population characteristics, underlying renal disease, time from presentation until dialysis, pre-dialysis risk factors, initial mode of dialysis, duration of renal replacement therapy, and cause of death were documented. Patients were studied until the end of November 1986 providing a minimum follow-up of 12 months, and maximum follow-up of 126 months for the elderly patients, and 151 months for those aged less than 60 years at start of treatment.

At the end of the follow-up period, 62 elderly patients were still alive. A questionnaire was sent to all of these survivors, designed to examine their social circumstances, their degree of independence, and their satisfaction with life, health and renal replacement therapy. Fifty six completed questionnaires $(90 \%)$ were returned; 2 patients died after the study period, before completing a questionnaire.

Cumulative survival of patients was calculated using actuarial analysis. Comparison between two proportions in Tables I and III was performed using the Chi-square tests. $P$ values of $<0.05$ were taken to indicate statistical significance. Means are expressed \pm standard deviation.

\section{Results}

One hundred and twenty two elderly people commenced renal replacement therapy in Newcastle during the period of study; $98(80 \%)$ of the patients were married, $7(6 \%)$ were single, $16(13 \%)$ were widowed, and 1 (1\%) was divorced. The majority of them were either retired $(67 \%)$ or housewives $(21 \%)$. Only $14(12 \%)$ were still in either full-time or part-time employment at the time of starting treatment.

Newcastle is responsible for providing a nephrological service for a large geographical area. Although $57 \%$ of the elderly patients lived in the Tyne and Wear area, other patients had to travel to Newcastle for dialysis or clinic appointments from Cumbria (18\%), Northumbria (16\%) and Co. Durham $(10 \%)$.

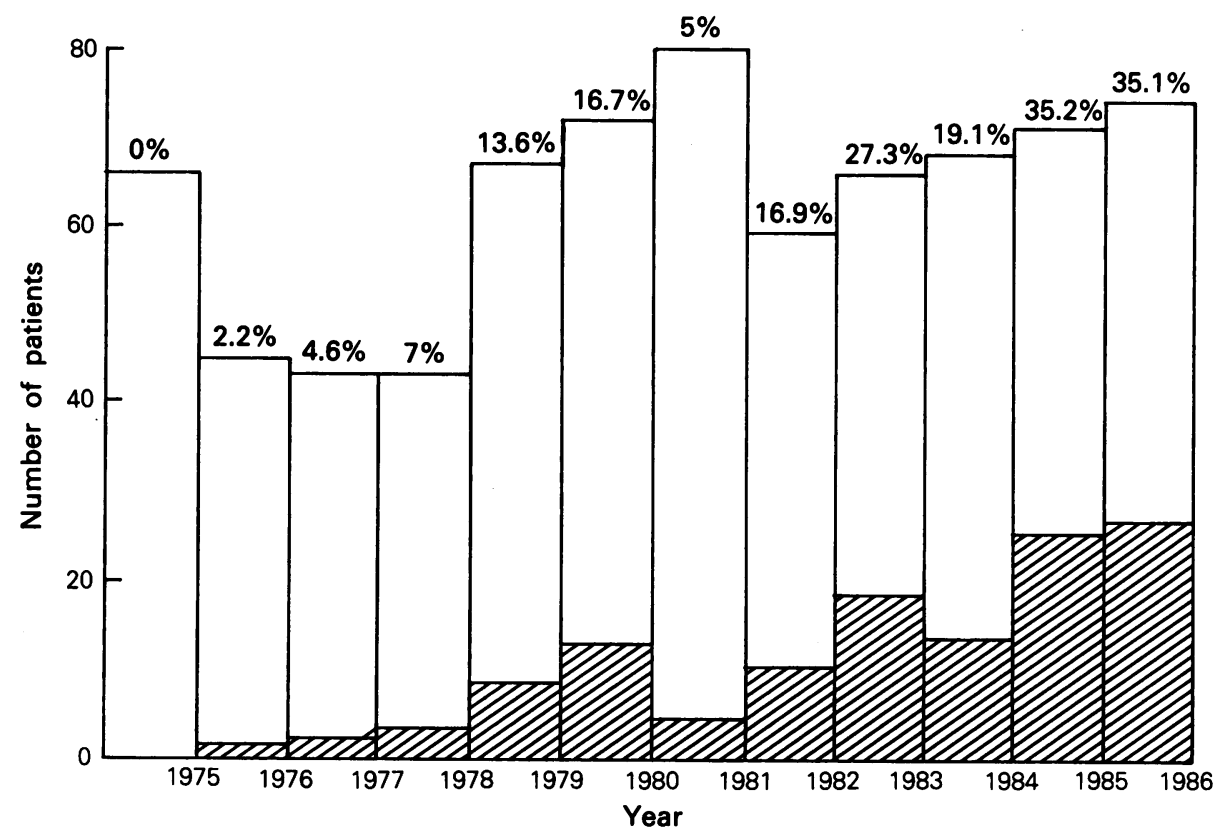

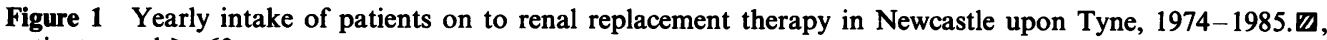
patients aged $>60$ years. 
Table I Causes of end-stage renal failure

\begin{tabular}{lccc}
\hline & $\begin{array}{c}\text { Age }<60 \text { years } \\
(n=632)\end{array}$ & $\begin{array}{c}\text { Age }>60 \text { years } \\
(n=122)\end{array}$ & $P$ \\
\cline { 1 - 2 } Glomerulonephritis & & $31(25 \%)$ & NS \\
Pyelonephritis & $187(30 \%)$ & & NS \\
Renovascular disease & $113(18 \%)$ & $16(13 \%)$ & NS \\
Polycystic kidney disease & $74(12 \%)$ & $13(11 \%)$ & NS \\
Diabetes mellitus & $66(10 \%)$ & $7(6 \%)$ & NS \\
Obstructive uropathy & $42(7 \%)$ & $4(13 \%)$ & $<0.001$ \\
Unknown & $3(0.5 \%)$ & $9(7 \%)$ & NS \\
\hline
\end{tabular}

Table I compares the cause of end-stage renal failure in the elderly patient population with the subjects aged below 60 years at the time of starting treatment. The pathology was similar in the two groups although obstructive uropathy was a significantly more common cause in the elderly patients.

The time from original presentation to the date of commencing renal replacement therapy in the elderly patients was 26.9 \pm 52.8 months (range 0-232 months). Table II lists the associated medical problems of the elderly subjects.

Figure 2 shows the number of patients aged more than 60 years accepted for renal replacement each year, along with the original form of dialysis used. A steady rise in the proportion of patients treated by CAPD is noted. All haemodialysis and intermittent peritoneal dialysis (IPD) has been performed in hospital. No elderly patient has begun treatment on home haemodialysis or home IPD.

Cumulative survival of elderly patients, and subjects aged less than 60 years at start of treatment is shown in Figure 3. At all time intervals the elderly patient had a lower survival. The five year survival of the elderly patient was $53 \%$, compared with $68 \%$ for the less than 60 year old individuals.

Thirteen of our cohort of 122 elderly patients

Table II Associated medical problems of elderly patients starting renal replacement therapy

\begin{tabular}{lr}
\hline & Number of patients $(\%)$ \\
\hline Hypertension & $54(44 \%)$ \\
Ischaemic heart disease & $26(21 \%)$ \\
Arthritis & $10(8 \%)$ \\
Carcinoma/myeloma & $8(7 \%)$ \\
Peptic ulceration & $7(6 \%)$ \\
Cerebro-vascular disease & $6(5 \%)$ \\
Obstructive airways disease & $6(5 \%)$ \\
Diabetes mellitus & $4(3 \%)$ \\
Hypothyroidism & $4(3 \%)$ \\
Gall stones & $3(2 \%)$ \\
Paraplegia & $1(1 \%)$ \\
Amyloidosis & $1(1 \%)$
\end{tabular}

underwent cadaveric renal transplantation. No living related transplants were performed, and no subject has received more than one graft. Mean age at transplantation was 63.7 years (range 62-67 years). Patients had been on dialysis for an average 22 months before grafting (range 5-46 months). Immunosuppression was with prednisolone and azathioprine for HLA matched kidneys $(n=10)$ and cyclosporin for ABO matched grafts $(n=3)$. Rejection episodes were treated with $500 \mathrm{mg}$ intravenous methylprednisolone on three successive days. The elderly patients have experienced between 0 and 4 rejection episodes each. One allograft never functioned and the patient returned to CAPD. Eight patients still have functioning grafts, on average 15 months after transplantation (range 7-32 months). Mean serum creatinine measured at their last clinic visit was $125 \mu \mathrm{mol} / 1$ (range 81-177 $\mu \mathrm{mol} / 1$ ). The remaining 4 transplanted elderly patients have died, on average 11 months after surgery (range 1-33 months). Causes of death were acute pancreatitis, vascular haemorrhage, cerebrovascular accident and myocardial infarction. All had functioning allografts at the time of death.

Table III documents the cause of death of 60 elderly patients ( $49 \%$ of cohort), and 246 subjects who were less than 60 years old at commencement of treatment (39\% of cohort). Cardiovascular disease was the commonest cause of death in both groups. Cessation of dialysis treatment was a significantly commoner cause of death in the elderly population $(P<0.01)$.

\section{Results of questionnaire}

Fifty six (36 males, 20 females) of the 62 elderly patients surviving at the end of the follow-up period returned completed questionnaires. Mean age of these patients was 67.8 years (range 61-78 years); 41 patients were married, 7 were widowed, 7 were single and 1 was divorced.

At the time of starting dialysis, only 5 of these patients were in full-time employment, and 1 worked part-time. Since starting dialysis, however, only one remains employed. 


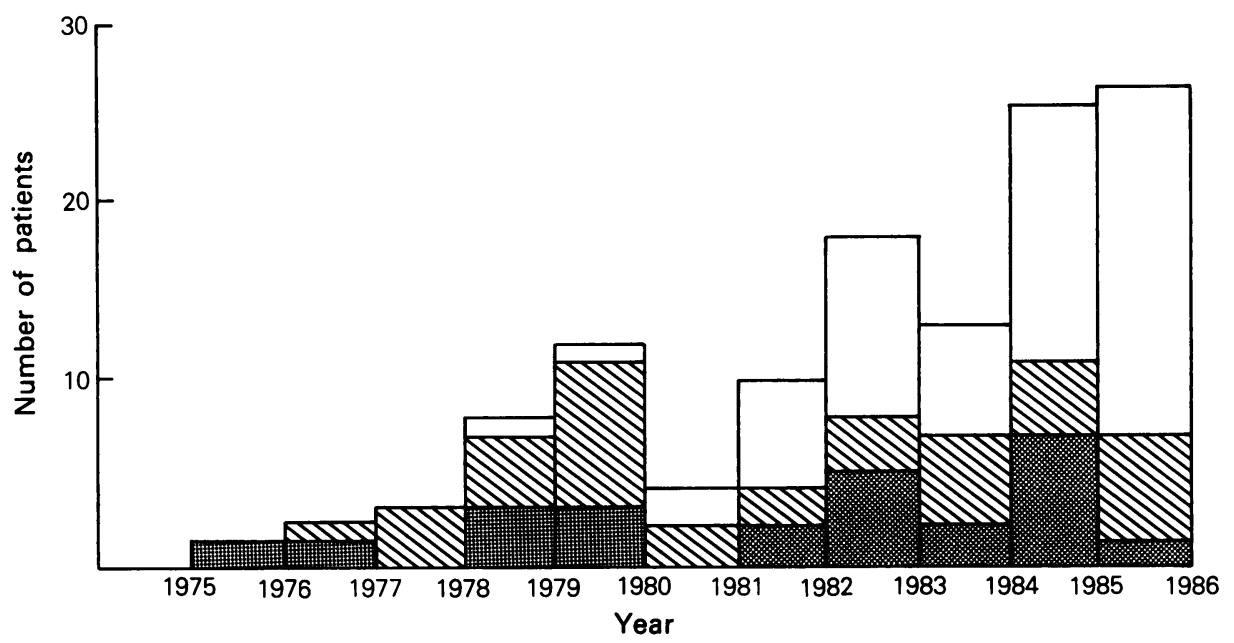

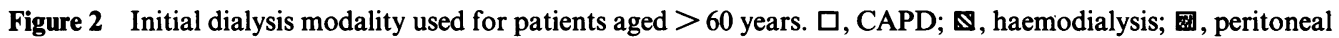
dialysis.

Fifty two $(93 \%)$ of these elderly patients lived in their own home, the remaining 4 stayed with a family member. No patient lived in residential care. Twenty five $(47 \%)$ remained totally independent, whereas 19 $(34 \%)$ required support from a relative, $10(18 \%)$ needed a home help, $8(14 \%)$ were regularly visited by a district nurse and $1(2 \%)$ patient received 'meals on wheels'.

Twenty patients $(36 \%)$ rarely left their home and considered themselves house-bound. All except 2 individuals were happy with the frequency of contact with relatives. Only 4 patients lack sufficient friends.

Table IV describes the evaluation of enjoyment of life, after Westlie et al. ${ }^{10}$ Although only $37 \%$ of those

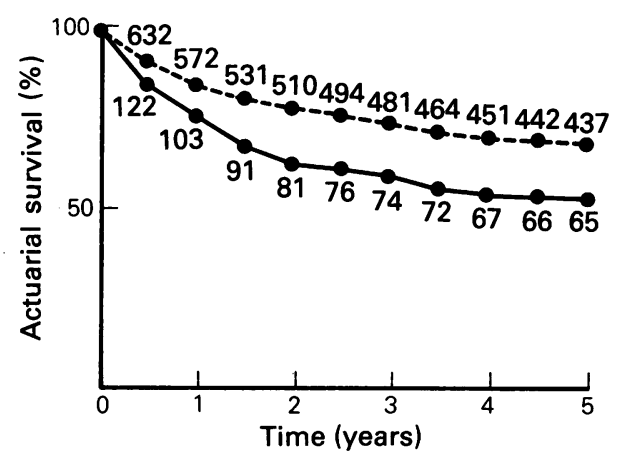

Figure 3 Cumulative survival on renal replacement therapy of patients aged $<60$ years $(0--0,(n=632)$ and patients aged $>60$ years $(\bigcirc-n=122)$. returning a questionnaire enjoyed life very much, it is reassuring to find that only two patients registered on the three lower scores of the scale.

All surviving elderly patients on renal replacement therapy were asked how they felt their health compared with others of their age. Although $45 \%$ felt less well, $21 \%$ felt the same, and surprisingly $34 \%$ actually felt they had better health.

Twenty three per cent of the elderly subjects had no complaints about their physical capabilities and felt they had normal physical activity; $50 \%$ felt they were able to carry out normal physical activity at least part of the time: $14 \%$ were only able to carry out physical activities involving self-care. The remaining $13 \%$, however, required assistance for care of bodily needs, and considered themselves to be debilitated.

Sixty three percent of the elderly patients who returned questionnaires travelled to hospital for dialysis or clinic appointments in ambulances or hospital cars; $34 \%$ used their own cars, whilst one patient travelled in a relative's car and one used public transport. One way travel took on average 55 minutes (range 5-210 minutes).

At the time of returning the questionnaires, renal replacement therapy was CAPD (28 patients), hospital haemodialysis (15), renal transplant (7) and hospital IPD (6). Although none of the 49 dialysis patients wanted to be treated by another dialysis technique, 30 of them $(61 \%)$ were keen to have a renal transplant. Only $6 \%$ had ever considered stopping treatment altogether.

All elderly patients returning a questionnaire were on medications: $55 \%$ took calcium supplements and $43 \%$ were on aluminium-containing phosphate bin- 
Table III Causes of death

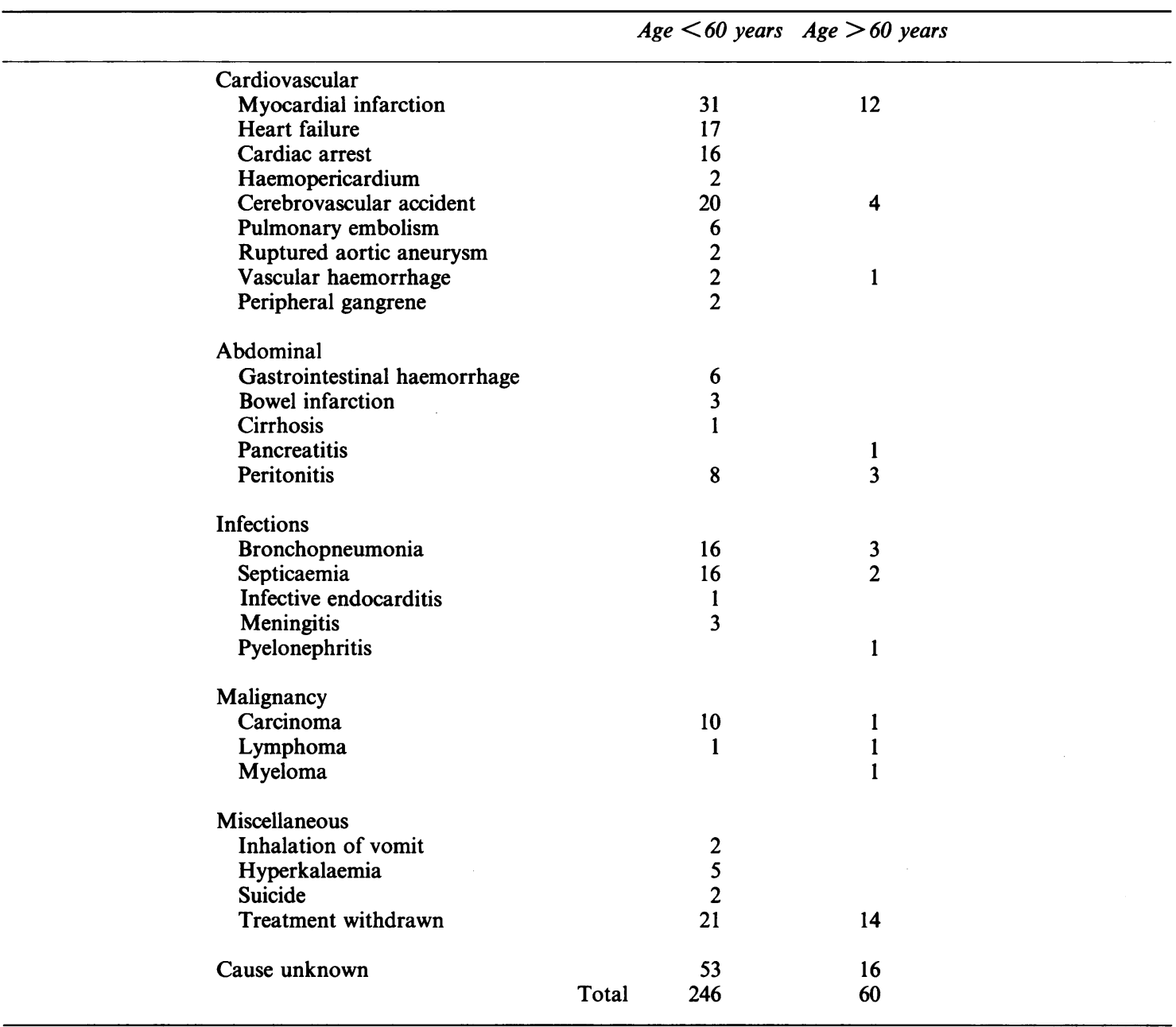

ders, $46 \%$ were taking antihypertensive therapy. Average number of medications per patient was 4.0 (range 1-9).

Table IV Enjoyment of life

\begin{tabular}{lc}
\hline Scale $^{*}$ & Number of patients (\%) \\
\hline 0 & $0(0 \%)$ \\
1 & $1(2 \%)$ \\
2 & $1(2 \%)$ \\
3 & $11(20 \%)$ \\
4 & $14(25 \%)$ \\
5 & $8(14 \%)$ \\
6 & $21(37 \%)$ \\
\hline
\end{tabular}

*Scale: $0=$ definitely not; $6=$ very much

\section{Discussion}

An increasing number of elderly patients with ESRF are now accepted for renal replacement therapy in Newcastle upon Tyne. In 1985 over one third of all subjects commencing treatment were over the age of 60 years. Indeed, it is our current policy to accept any patient who is potentially rehabilitable for maintenance dialysis, regardless of age. Only metastatic cancer and senile dementia remain contraindications.

This liberal acceptance policy, however, has to be balanced against increasing financial constraints. It is difficult to know which mode of renal replacement therapy is best for the elderly patient, in the absence of randomized, prospective studies. Until recently, the vast majority have been treated with hospital haemodialysis. ${ }^{4-10}$ However, this form of treatment is 
the most expensive option, and as there has been very little expansion of hospital haemodialysis facilities in Britain in recent years, few spaces are available for elderly patients. No patient beginning treatment after the age of 60 has started on home haemodialysis in Newcastle. Previous reports have suggested that geriatric patients are not suitable for home haemodialysis. ${ }^{13,14}$ However, one third of a group of over-70 year olds with ESRF were satisfactorily treated with home haemodialysis by Westlie et al. ${ }^{10}$ although all but one needed an attendant. As most of our patients are married, this alternative merits future consideration. Only 13 of our elderly patients received a kidney transplant. However, almost two thirds of elderly dialysis patients returning the questionnaires were keen to have a transplant. Results of renal transplantation were generally good, with a cumulative patient survival rate of $74 \%$ at 2 years after surgery. As elderly people with ESRF are unlikely to have a suitable living-related donor, and as cadaveric organs are preferentially given to younger recipients, this treatment option does not provide the answer for the elderly patient with ESRF at present.

The introduction of CAPD into Newcastle in 1978 has been primarily responsible for our liberal acceptance of elderly people on to dialysis. Indeed, it is currently our treatment of first choice for such patients. In 1985, 73\% of patients over 60 years of age with ESRF began treatment with CAPD (Figure 2). CAPD offers many benefits, but the absence of long journeys to and from hospital for dialysis is particularly attractive to the older patient. Forty four percent of our total elderly patient cohort lived away from the Newcastle area. The peritonitis rate of elderly CAPD patients does not appear to be greater than younger subjects. ${ }^{11}$ Mortality from CAPD peritonitis, however, appears to rise with age. ${ }^{15}$ CAPD also has a high 'drop-out' rate ${ }^{16}$ which places further demands on overburdened hospital haemodialysis units.

An increase in government funding of renal services and expansion of hospital haemodialysis facilities appears essential. Our study suggests that once accepted for dialysis, the elderly patient with ESRF can expect a 5 year survival rate of $53 \%$. This figure is similar to that reported by others, ${ }^{2,7}$ and compares very favourably with the survival of patients with certain malignant tumours whose very costly aggressive treat- ment suffers little criticism. ${ }^{7}$

Many of our elderly patients with ESRF had associated medical problems at the time of starting treatment (Table II). It is, however, not possible to analyse such factors from our data to identify patients who are poor risks for dialysis. Westlie et al. ${ }^{10}$ suggested that arteriosclerotic heart disease and year of entry were significant risk factors. Indeed, the commonest causes of death in our series were cardiovascular diseases (Table III), a fact also reported by other workers. ${ }^{7,9}$

Quality of life on renal replacement therapy is as important as prognosis when considering an elderly person for treatment. Our study indicates that these patients usually enjoy life and are happy with their method of dialysis. They generally live at home $(50 \%$ being totally independent) and have frequent contact with family and friends. The majority were satisfied with their health on renal replacement therapy, and most are active with good physical capabilities.

However, the results of treating elderly patients with ESRF are not entirely satisfactory. It is disappointing that although $60 \%$ of our dialysis patients who returned questionnaires were keen to have a kidney transplant, there is little realistic chance of them receiving one. A third of our patients are house-bound and a significant minority consider themselves to be debilitated. Indeed, three patients have been so unwell on dialysis they have considered discontinuing treatment altogether. In addition, cessation of treatment was responsible for one quarter of all deaths in our elderly patient population so that our quality of life survey is, to some extent, not representative since, of necessity, it includes only survivors.

The results of this study support the view that patients over 60 years old should not be denied treatment for ESRF solely because of their age. Elderly people generally do well on renal replacement therapy, with good survival and quality of life. it is unfortunately difficult to identify, before treatment has started, the patients who will do poorly. A 'trial of dialysis' may therefore be required. Acceptance of increasing numbers of elderly patients, however, requires greater funding of renal services with expansion of hospital haemodialysis facilities, in particular, to provide a back-up for those individuals who are treatment failures on CAPD.

\section{References}

1. Wing, A.J., Brunner, F.P., Brynger, H. et al. Combined report on regular dialysis and transplantation in Europe. Proc Eur Dial Transplant Assoc Eur Ren Assoc 1977, 15: 4-76.

2. Taube, D.H., Winder, E.A., Ogg, C.S. et al. Successful treatment of middle aged and elderly patients with end-

stage renal disease. $\mathrm{Br}$ Med J 1983, 286: 2018-2020.

3. Berlyne, G.M. Over 50 and uremic = death. Nephron 1982, 31: 189-192.

4. Figueroa, J.E. Management of uremia in older patients: haemodialysis and renal transplantation. J Am Geriatric Soc 1968, 16: 1323-1330. 
5. Ghantous, W.N., Bailey, G.L., Zschaek, D., Hampers, C.L. \& Merril, J.P. Long-term haemodialysis in the elderly. Trans Am Soc Artif Intern Organs 1971, 17: 125128.

6. Cohen, S.L., Comty, C.M. \& Shapiro, F.L. The effect of age on the results of regular hemodialysis treatment. Proc Eur Dial Transplant Assoc Eur Ren Assoc 1970, 7: 254-260.

7. Jacobs, C., Diallo, A., Balas, E.A., Nectoux, M. \& Etienne, $S$. Maintenance haemodialysis treatment in patients aged over 60 years. Demographic profile, clinical aspects and outcome. Proc Eur Dial Transplant Assoc Eur Ren Assoc 1984, 21: 477-489.

8. Mion, C., Oules, R., Canaud, P. et al. Maintenance dialysis in the elderly. A review of 15 years experience in Languedoc and Rousillon. Proc Eur Dial Transplant Assoc Eur Ren Assoc 1984, 21: 490-509.

9. Schaefer, K., Asmus, G., Quellhorst, E., Pauls, A., von Herrath, D. \& Jaunke, J. Optimum dialysis treatment for patients over 60 years with primary renal disease. Survival data and clinical results from 242 patients treated either by haemodialysis or haemofiltration. Proc Eur Dial Transplant Assoc Eur Ren Assoc 1984, 21: 510517.
10. Westlie, L., Umen, A., Nestrud, S. \& Kjellstrand, C.M. Mortality, morbidity and life satisfaction in the very old dialysis patient. Trans Am Soc Artif Intern Organs 1984, 30: $21-29$.

11. Nicholls, A.J., Waldek, S., Platts, M.M., Moorhead, P.J. \& Brown, C.B. Impact of continuous ambulatory peritoneal dialysis on treatment of renal failure in patients aged over 60. Br Med J 1984, 288: 18-19.

12. Kaye, M., Patel, P.A. \& Sommerville, P.S. Four years experience with CAPD in the elderly. Perit Dial Bull 1983, 3: 17-19.

13. Johnson, J.P., McCauley, C.R. \& Copley, J.B. The quality of life of haemodialysis and transplant patients. Kidney Int 1982, 22: 286-291.

14. Evans, R.W. \& Bryan, F.A. The renal dialysis study: II Demographic description of the patient sample. Dial Transplant 1980, 9: 1173-1176.

15. Fenton, S.S. Peritonitis-related deaths among CAPD patients. Perit Dial Bull 1983, 3: S9-S11.

16. Heaton, A., Rodgers, R.S.C., Sellars, L. et al. Continuous ambulatory peritoneal dialysis after the honeymoon: review of experience in Newcastle 1979-84. $\mathrm{Br}$ Med J 1986, 293: 938-941. 